\title{
On Generalizations of Ostrowski Inequality via Euler Harmonic Identities
}

\author{
L. J. DEDIĆa,*, M. MATIĆb,†, J. PEČARIĆc, ${ }^{c, \ddagger}$ and A. VUKELIĆ
}

${ }^{a}$ Department of Mathematics, University of Split, Teslina 12, 21000 Split, Croatia; ${ }^{\mathrm{b}}$ FESB, Mathematics Department, University of Split, R. Boškovića

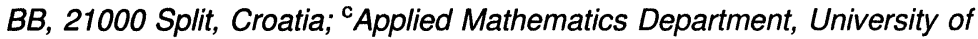
Adelaide, Australia; ${ }^{\mathrm{d}}$ Faculty of Food Technology and Biotechnology, Mathematics Department, University of Zagreb, Pierottijeva 6, 10000 Zagreb, Croatia

(Received 7 April 2001; Revised 10 July 2001)

Some generalizations of Ostrowski inequality are given, by using some Euler identities involving harmonic sequences of polynomials.

Keywords: Ostrowski inequality; Harmonic polynomials

Classification: 1991 Mathematics Subject Classification: 26D15, 26D20, 26D99

\section{INTRODUCTION}

Let $B_{k}(t), k \geq 0$, be the Bernoulli polynomials, $B_{k}=B_{k}(0), k \geq 0$, the Bernoulli numbers. The Bernoulli polynomials $B_{k}(t), k \geq 0$ are uniquely determined by the following identities

$$
B_{k}^{\prime}(t)=k B_{k-1}(t), \quad k \geq 1 ; \quad B_{0}(t)=1
$$

* E-mail: ljuban@pmfst.hr

† Corresponding author. E-mail: mmatic@fesb.hr

‡ E-mail: jpecaric@spam.adelaide.edu.au

"E-mail: avukelic@pbf.hr 
and

$$
B_{k}(t+1)-B_{k}(t)=k t^{k-1}, \quad k \geq 0 .
$$

For example, the three first Bernoulli polynomials are given by $B_{0}(t)=1, B_{1}(t)=t-(1 / 2), B_{2}(t)=t^{2}-t+(1 / 6)$. For some further details on the Bernoulli polynomials and the Bernoulli numbers see for example [1] or [2].

Let $B_{k}^{*}(t), k \geq 0$, be the periodic functions of period 1, related to the Bernoulli polynomials as

$$
B_{k}^{*}(t)=B_{k}(t), \quad 0 \leq t<1, \quad B_{k}^{*}(t+1)=B_{k}^{*}(t), \quad t \in \mathbf{R} .
$$

From the properties of the Bernoulli polynomials it follows that $B_{0}^{*}=1, B_{1}^{*}$ is a discontinuous function with a jump of -1 at each integer, and $B_{k}^{*}, k \geq 2$, is a continuous function.

Let $f:[a, b] \rightarrow \mathbf{R}$ be such that $f^{(n-1)}$ is a function of bounded variation on $[a, b]$ for some $n \geq 1$. In the recent paper [4] the following two identities have been proved:

$$
f(x)=\frac{1}{b-a} \int_{a}^{b} f(t) \mathrm{d} t+T_{n}(x)+R_{n}^{1}(x)
$$

and

$$
f(x)=\frac{1}{b-a} \int_{a}^{b} f(t) \mathrm{d} t+T_{n-1}(x)+R_{n}^{2}(x)
$$

where $T_{0}(x)=0$ and

$$
T_{m}(x)=\sum_{k=1}^{m} \frac{(b-a)^{k-1}}{k !} B_{k}\left(\frac{x-a}{b-a}\right)\left[f^{(k-1)}(b)-f^{(k-1)}(a)\right],
$$

for $1 \leq m \leq n$, while

$$
R_{n}^{1}(x)=-\frac{(b-a)^{n-1}}{n !} \int_{[a, b]} B_{n}^{*}\left(\frac{x-t}{b-a}\right) \mathrm{d} f^{(n-1)}(t)
$$


and

$$
R_{n}^{2}(x)=-\frac{(b-a)^{n-1}}{n !} \int_{[a, b]}\left[B_{n}^{*}\left(\frac{x-t}{b-a}\right)-B_{n}\left(\frac{x-a}{b-a}\right)\right] \mathrm{d} f^{(n-1)}(t) .
$$

Here, as in the rest of the paper, we write $\int_{[a, b]} g(t) \mathrm{d} \varphi(t)$ to denote the Riemann-Stieltjes integral with respect to a function $\varphi:[a, b] \rightarrow \mathbf{R}$ of bounded variation, and $\int_{a}^{b} g(t) \mathrm{d} t$ for the Riemann integral. The formulae (1.3) and (1.4) hold for every $x \in[a, b]$. They are extensions of the well known formula for the expansion of an arbitrary function $f$ with a continuous $n$th derivative $f^{(n)}$ in Bernoulli polynomials [3, p. 17]:

$$
f(x)=\frac{1}{b-a} \int_{a}^{b} f(t) \mathrm{d} t+T_{n-1}(x)+R_{n}(x)
$$

where

$$
R_{n}(x)=-\frac{(b-a)^{n-1}}{n !} \int_{a}^{b}\left[B_{n}^{*}\left(\frac{x-t}{b-a}\right)-B_{n}\left(\frac{x-a}{b-a}\right)\right] f^{(n)}(t) \mathrm{d} t .
$$

Let the polynomials $P_{k}(t), k \geq 0$ satisfy the following condition

$$
P_{k}^{\prime}(t)=P_{k-1}(t), \quad k \geq 1 ; \quad P_{0}(t)=1
$$

For a sequence $\left(P_{k}(t), k \geq 0\right)$ of polynomials satisfying the condition (1.5), we say that it is a harmonic sequence of polynomials. From (1.5), by an easy induction it follows that every harmonic sequence of polynomials must be of the form

$$
P_{k}(t)=\sum_{i=0}^{k} \frac{c_{i}}{(k-i) !} t^{k-i}, \quad k \geq 0
$$

where $\left(c_{k}, k \geq 0\right)$ is a sequence of real numbers such that $c_{0}=1$. In fact, $c_{k}=P_{k}(0), k \geq 0$. Especially, we have $P_{0}(t)=1, P_{1}(t)=t+c_{1}$, $P_{2}(t)=(1 / 2) t^{2}+c_{1} t+c_{2}$.

The aim of this paper is to generalize the formulae (1.3) and (1.4), by replacing the Bernoulli polynomials by an arbitrary harmonic sequence 
of polynomials, and using them to prove some generalizations of Ostrowski inequality.

\section{EULER HARMONIC IDENTITIES}

Assume that $\left(P_{k}(t), k \geq 0\right)$ is a harmonic sequence of polynomials i.e. the sequence of polynomials satisfying the condition (1.5). Define $P_{k}^{*}(t), k \geq 0$ to be a periodic functions of period 1 , related to $P_{k}(t)$, $k \geq 0$ as

$$
P_{k}^{*}(t)=P_{k}(t), \quad 0 \leq t<1, \quad P_{k}^{*}(t+1)=P_{k}^{*}(t), \quad t \in \mathbf{R} .
$$

Thus, $P_{0}^{*}(t)=1$, while for $k \geq 1, P_{k}^{*}(t)$ is continuous on $\mathbf{R} \backslash \mathbf{Z}$ and has a jump of

$$
\alpha_{k}=P_{k}(0)-P_{k}(1)
$$

at every integer $t$, whenever $\alpha_{k} \neq 0$. Note that $\alpha_{1}=-1$, since $P_{1}(t)=t+c_{1}$, for some $c_{1} \in \mathbf{R}$. Also, note that from (1.5) it follows

$$
P_{k}^{* \prime}(t)=P_{k-1}^{*}(t), \quad k \geq 1, \quad t \in \mathbf{R} \backslash \mathbf{Z} .
$$

Let $a, b \in \mathbf{R}, a<b$, and $f:[a, b] \rightarrow \mathbf{R}$ be such that $f^{(n-1)}$ is a function of bounded variation on $[a, b]$ for some $n \geq 1$. For every $x \in[a, b]$ and $1 \leq m \leq n$ we introduce the following notations

$$
\tilde{T}_{m}(x)=\sum_{k=1}^{m}(b-a)^{k-1} P_{k}\left(\frac{x-a}{b-a}\right)\left[f^{(k-1)}(b)-f^{(k-1)}(a)\right],
$$

with convention $\tilde{T}_{0}(x)=0$, and

$$
\tau_{m}(x)=\sum_{k=2}^{m}(b-a)^{k-1} \alpha_{k} f^{(k-1)}(x),
$$

with convention $\tau_{1}(x)=0$. 
LeMma 1 Let $a, b \in \mathbf{R}, a<b, x \in[a, b], k \geq 0$. Define $\varphi_{k}(x ; \cdot)$ : $[a, b] \rightarrow \mathbf{R}$ as

$$
\varphi_{k}(x ; t)=P_{k}^{*}\left(\frac{x-t}{b-a}\right), \quad a \leq t \leq b .
$$

If $k \geq 1$, then for every continuous function $F:[a, b] \rightarrow \mathbf{R}$, we have

$$
\int_{[a, b]} F(t) \mathrm{d} \varphi_{k}(x ; t)=-\frac{1}{b-a} \int_{a}^{b} F(t) \varphi_{k-1}(x ; t) \mathrm{d} t-\alpha_{k} F(x),
$$

for $a \leq x<b$, and

$$
\int_{[a, b]} F(t) \mathrm{d} \varphi_{k}(b ; t)=-\frac{1}{b-a} \int_{a}^{b} F(t) \varphi_{k-1}(b ; t) \mathrm{d} t-\alpha_{k} F(a) .
$$

Proof Let $k \geq 1$ and assume that $a<x<b$. The function $\varphi_{k}(x ; \cdot)$ is differentiable on $[a, b] \backslash\{x\}$ and its derivative is equal to $(-1 /$ $(b-a)) \varphi_{k-1}(x ; \cdot)$, by (2.2). Further, it has a jump of $\varphi_{k}(x ; x+0)-$ $\varphi_{k}(x ; x-0)=-\alpha_{k}$ at $x$, which gives the first formula in this case. For $x=a$ the function $\varphi_{k}(a ; \cdot)$ is differentiable on $(a, b)$ and its derivative is equal to $(-1 /(b-a)) \varphi_{k-1}(a ; \cdot)$. Further, it has jump of $\varphi_{k}(a ; a+0)-$ $\varphi_{k}(a ; a)=-\alpha_{k}$ at the point $a$, while $\varphi_{k}(a ; b)-\varphi_{k}(a ; b-0)=0$, which gives the first formula for $x=a$. The second formula is a consequence of the first one and of the fact that $\varphi_{k}(b ; \cdot)=\varphi_{k}(a ; \cdot)$.

THEOREM 1 Let $\left(P_{k}, k \geq 0\right)$ be a harmonic sequence of polynomials and $f:[a, b] \rightarrow \mathbf{R}$ such that $f^{(n-1)}$ is a continuous function of bounded variation on $[a, b]$ for some $n \geq 1$. Then for every $x \in[a, b]$

$$
\begin{aligned}
& f(x)=\frac{1}{b-a} \int_{a}^{b} f(t) \mathrm{d} t+\tilde{T}_{n}(x)+\tau_{n}(x)+\tilde{R}_{n}^{1}(x), \\
& f(x)=\frac{1}{b-a} \int_{a}^{b} f(t) \mathrm{d} t+\tilde{T}_{n-1}(x)+\tau_{n}(x)+\tilde{R}_{n}^{2}(x),
\end{aligned}
$$


where $\tilde{T}_{n}(x)$ and $\tau_{n}(x)$ are defined by (2.3) and (2.4), respectively, and

$$
\begin{aligned}
& \tilde{R}_{n}^{1}(x)=-(b-a)^{n-1} \int_{[a, b]} P_{n}^{*}\left(\frac{x-t}{b-a}\right) \mathrm{d} f^{(n-1)}(t), \\
& \tilde{R}_{n}^{2}(x)=-(b-a)^{n-1} \int_{[a, b]}\left[P_{n}^{*}\left(\frac{x-t}{b-a}\right)-P_{n}\left(\frac{x-a}{b-a}\right)\right] \mathrm{d} f^{(n-1)}(t) .
\end{aligned}
$$

Proof For $1 \leq k \leq n$ consider the integrals

$$
I_{k}(x)=(b-a)^{k-1} \int_{[a, b]} P_{k}^{*}\left(\frac{x-t}{b-a}\right) \mathrm{d} f^{(k-1)}(t) .
$$

By partial integration we get

$$
\begin{aligned}
I_{k}(x)= & \left.(b-a)^{k-1} P_{k}^{*}\left(\frac{x-t}{b-a}\right) f^{(k-1)}(t)\right|_{a} ^{b} \\
& -(b-a)^{k-1} \int_{[a, b]} f^{(k-1)}(t) \mathrm{d} P_{k}^{*}\left(\frac{x-t}{b-a}\right) .
\end{aligned}
$$

First, assume that $a \leq x<b$. For every $k \geq 1$ we have

$$
P_{k}^{*}\left(\frac{x-b}{b-a}\right)=P_{k}^{*}\left(\frac{x-a}{b-a}-1\right)=P_{k}^{*}\left(\frac{x-a}{b-a}\right)=P_{k}\left(\frac{x-a}{b-a}\right) .
$$

Therefore, using the first formula from Lemma 1, we get from (2.7)

$$
\begin{aligned}
I_{k}(x)= & (b-a)^{k-1} P_{k}\left(\frac{x-a}{b-a}\right)\left[f^{(k-1)}(b)-f^{(k-1)}(a)\right]+(b-a)^{k-1} \\
& \times \alpha_{k} f^{(k-1)}(x)+(b-a)^{k-2} \int_{a}^{b} f^{(k-1)}(t) P_{k-1}^{*}\left(\frac{x-t}{b-a}\right) \mathrm{d} t .
\end{aligned}
$$

Since $\alpha_{1}=-1$, for $k=1(2.8)$ reduces to

$$
I_{1}(x)=P_{1}\left(\frac{x-a}{b-a}\right)[f(b)-f(a)]-f(x)+\frac{1}{b-a} \int_{a}^{b} f(t) \mathrm{d} t
$$


For $k \geq 2$ we have

$$
\begin{aligned}
& (b-a)^{k-2} \int_{a}^{b} f^{(k-1)}(t) P_{k-1}^{*}\left(\frac{x-t}{b-a}\right) \mathrm{d} t \\
& \quad=(b-a)^{k-2} \int_{[a, b]} P_{k-1}^{*}\left(\frac{x-t}{b-a}\right) \mathrm{d} f^{(k-2)}(t)=I_{k-1}(x)
\end{aligned}
$$

and (2.8) can be rewritten as

$$
\begin{aligned}
I_{k}(x)= & (b-a)^{k-1} P_{k}\left(\frac{x-a}{b-a}\right)\left[f^{(k-1)}(b)-f^{(k-1)}(a)\right] \\
& +(b-a)^{k-1} \alpha_{k} f^{(k-1)}(x)+I_{k-1}(x) .
\end{aligned}
$$

From (2.9) and (2.10) it is easy to obtain

$$
\begin{aligned}
I_{n}(x)= & \sum_{k=1}^{n}(b-a)^{k-1} P_{k}\left(\frac{x-a}{b-a}\right)\left[f^{(k-1)}(b)-f^{(k-1)}(a)\right] \\
& +\sum_{k=2}^{n}(b-a)^{k-1} \alpha_{k} f^{(k-1)}(x)-f(x)+\frac{1}{b-a} \int_{a}^{b} f(t) \mathrm{d} t,
\end{aligned}
$$

which is equivalent to (2.5), since $I_{n}(x)=-\tilde{R}_{n}^{1}(x)$. Thus, (2.5) holds for $a \leq x<b$. If $x=b$, then we have $P_{k}^{*}((b-b) /(b-a))=P_{k}^{*}(0)=$ $P_{k}(0), P_{k}^{*}((b-a) /(b-a))=P_{k}^{*}(1)=P_{k}^{*}(0)=P_{k}(0)$. Similarly as we did for $a \leq x<b$, using the above equalities and the second formula from Lemma 1, we get from (2.7).

$$
\begin{aligned}
I_{k}(b)= & (b-a)^{k-1} P_{k}(0)\left[f^{(k-1)}(b)-f^{(k-1)}(a)\right] \\
& +(b-a)^{k-1} \alpha_{k} f^{(k-1)}(a)+I_{k-1}(b),
\end{aligned}
$$

for $k \geq 2$, and $I_{1}(b)=P_{1}(0)[f(b)-f(a)]-f(a)+(1 /(b-a)) \int_{a}^{b} f(t) \mathrm{d} t$. Applying the above identities, we get

$$
\begin{aligned}
I_{n}(b)= & \sum_{k=1}^{n}(b-a)^{k-1} P_{k}(0)\left[f^{(k-1)}(b)-f^{(k-1)}(a)\right] \\
& +\sum_{k=2}^{n}(b-a)^{k-1} \alpha_{k} f^{(k-1)}(a)-f(a)+\frac{1}{b-a} \int_{a}^{b} f(t) \mathrm{d} t .
\end{aligned}
$$


We have $\alpha_{1}=-1$ and, by $(2.1), P_{k}(0)=P_{k}(1)+\alpha_{k}$. Therefore, the last identity can be rewritten as

$$
\begin{aligned}
I_{n}(b)= & \sum_{k=1}^{n}(b-a)^{k-1} P_{k}(1)\left[f^{(k-1)}(b)-f^{(k-1)}(a)\right] \\
& +\sum_{k=2}^{n}(b-a)^{k-1} \alpha_{k} f^{(k-1)}(b)-f(b)+\frac{1}{b-a} \int_{a}^{b} f(t) \mathrm{d} t,
\end{aligned}
$$

which is equivalent to (2.5) for $x=b$, since $I_{n}(b)=-\tilde{R}_{n}^{1}(b)$. Note that

$$
\begin{aligned}
\tilde{R}_{n}^{2}(x) & =\tilde{R}_{n}^{1}(x)+(b-a)^{n-1} P_{n}\left(\frac{x-a}{b-a}\right) \int_{[a, b]} \mathrm{d} f^{(n-1)}(t) \\
& =\tilde{R}_{n}^{1}(x)+(b-a)^{n-1} P_{n}\left(\frac{x-a}{b-a}\right)\left[f^{(n-1)}(b)-f^{(n-1)}(a)\right] \\
& =\tilde{R}_{n}^{1}(x)+\tilde{T}_{n}(x)-\tilde{T}_{n-1}(x) .
\end{aligned}
$$

Therefore, $\tilde{R}_{n}^{1}(x)=-\tilde{T}_{n}(x)+\tilde{T}_{n-1}(x)+\tilde{R}_{n}^{2}(x)$, so that the formula $(2.6)$ follows from the formula (2.5).

Example 1 Let $P_{k}(t)=(1 / k !) B_{k}(t), k \geq 0$, where $B_{k}(t)$ are the Bernoulli polynomials. From (1.1) it follows that $\left(P_{k}(t), k \geq 0\right)$ is a harmonic sequence of polynomials. Also, we have $[1,23.1 .19,23.1 .20]$

$$
B_{2 j}(0)=B_{2 j}(1)=B_{2 j}, \quad B_{2 j+1}(0)=B_{2 j+1}(1)=0, \quad j \geq 1,
$$

which implies that $\alpha_{k}=0$ for $k \geq 2$, while $\alpha_{1}=-1$ as in the general case. Moreover, in this case, for any $f:[a, b] \rightarrow \mathbf{R}$ such that $f^{(n-1)}$ is a continuous function of bounded variation on $[a, b]$, we get

$\tilde{T}_{m}(x)=T_{m}(x), \tau_{m}(x)=0, \quad m \leq n$ and $\tilde{R}_{n}^{1}(x)=R_{n}^{1}(x), \quad \tilde{R}_{n}^{2}(x)=R_{n}^{2}(x)$,

where $T_{m}(x), R_{n}^{1}(x)$ and $R_{n}^{2}(x)$ are defined as in the Introduction. Consequently, the formulae (2.5) and (2.6) become (1.3) and (1.4), respectively.

Example 2 For fixed $\gamma \in \mathbf{R}$ define

$$
P_{k}(t)=\frac{1}{k !}(t-\gamma)^{k}, \quad k \geq 0 .
$$


Then $\left(P_{k}(t), k \geq 0\right)$ is a harmonic sequence of polynomials, and

$$
P_{k}(0)=\frac{1}{k !}(-\gamma)^{k}, \quad P_{k}(1)=\frac{1}{k !}(1-\gamma)^{k} .
$$

Therefore, in this case

$$
\alpha_{k}=P_{k}(0)-P_{k}(1)=\frac{1}{k !}\left[(-\gamma)^{k}-(1-\gamma)^{k}\right], \quad k \geq 1 .
$$

Further, we have

$$
\tilde{T}_{m}(x)=\sum_{k=1}^{m} \frac{(b-a)^{k-1}}{k !}\left(\frac{x-a}{b-a}-\gamma\right)^{k}\left[f^{(k-1)}(b)-f^{(k-1)}(a)\right]
$$

and

$$
\tau_{m}(x)=\sum_{k=2}^{m} \frac{(b-a)^{k-1}}{k !}\left[(-\gamma)^{k}-(1-\gamma)^{k}\right] f^{(k-1)}(x),
$$

for every $x \in[a, b]$.

\section{GENERALIZATIONS OF THE OSTROWSKI INEQUALITY}

In this section we shall use the same notations as above.

THEOREM 2 Suppose that $\left(P_{k}(t), k \geq 0\right)$ is a harmonic sequence of polynomials. Let $f:[a, b] \rightarrow \mathbf{R}$ be such that $f^{(n-1)}$ is an L-Lipschitzian function on $[a, b]$ for some $n \geq 1$. Then

$$
\begin{gathered}
\left|f(x)-\frac{1}{b-a} \int_{a}^{b} f(t) \mathrm{d} t-\tilde{T}_{n-1}(x)-\tau_{n}(x)\right| \\
\leq(b-a)^{n} \int_{0}^{1}\left|P_{n}(t)-P_{n}\left(\frac{x-a}{b-a}\right)\right| \mathrm{d} t \cdot L \\
\left|f(x)-\frac{1}{b-a} \int_{a}^{b} f(t) \mathrm{d} t-\tilde{T}_{n}(x)-\tau_{n}(x)\right| \leq(b-a)^{n} \int_{0}^{1}\left|P_{n}(t)\right| \mathrm{d} t \cdot L
\end{gathered}
$$

for every $x \in[a, b]$. 
Proof If $\varphi:[a, b] \rightarrow \mathbf{R}$ is $L$-Lipschitzian on $[a, b]$, that is $|\varphi(x)-\varphi(y)| \leq L \cdot|x-y|, \quad \forall x, y \in[a, b]$, then for any integrable function $g:[a, b] \rightarrow \mathbf{R}$ we have

$$
\left|\int_{[a, b]} g(t) \mathrm{d} \varphi(t)\right| \leq \int_{a}^{b}|g(t)| \mathrm{d} t \cdot L .
$$

Using this estimate we get

$$
\begin{aligned}
\left|\tilde{R}_{n}^{2}(x)\right| & =(b-a)^{n-1}\left|\int_{[a, b]}\left[P_{n}^{*}\left(\frac{x-t}{b-a}\right)-P_{n}\left(\frac{x-a}{b-a}\right)\right] \mathrm{d} f^{(n-1)}(t)\right| \\
& \leq(b-a)^{n-1} \int_{a}^{b}\left|P_{n}^{*}\left(\frac{x-t}{b-a}\right)-P_{n}\left(\frac{x-a}{b-a}\right)\right| \mathrm{d} t \cdot L .
\end{aligned}
$$

Since the function $P_{n}^{*}(\cdot)$ has period 1 , we have

$$
\int_{0}^{1}\left|P_{n}^{*}(y+t)-z\right| \mathrm{d} t=\int_{0}^{1}\left|P_{n}^{*}(t)-z\right| \mathrm{d} t=\int_{0}^{1}\left|P_{n}(t)-z\right| \mathrm{d} t,
$$

for every $y, z \in \mathbf{R}$. Therefore

$$
\int_{a}^{b}\left|P_{n}^{*}\left(\frac{x-t}{b-a}\right)-P_{n}\left(\frac{x-a}{b-a}\right)\right| \mathrm{d} t=(b-a) \int_{0}^{1}\left|P_{n}(t)-P_{n}\left(\frac{x-a}{b-a}\right)\right| \mathrm{d} t,
$$

which implies

$$
\left|\tilde{R}_{n}^{2}(x)\right| \leq(b-a)^{n} \int_{0}^{1}\left|P_{n}(t)-P_{n}\left(\frac{x-a}{b-a}\right)\right| \mathrm{d} t \cdot L
$$

and (3.1) follows from (2.6).

The inequality (3.2) follows from (2.5) by the similar argument.

Remark 1 Both of the above inequalities for $n=1$ (the second one with $c_{1}=-((x-a) /(b-a))$ are reduced to the Ostrowski inequality for a function $f$ which is $L$-Lipschitzian on $[a, b]$ (see [10]).

Remark 2 (i) The inequality (3.1) for $n=2$ and $c_{1}=(-1 / 2)$ was proved in [4]. Also it is an improvement and extension of the similar result from [13] (for details see Remark 3 in [4]). 
For $x=a$ or $x=b$ we have the trapezoid inequality which with its generalization and applications was considered in [5] (see also [16]).

For $x=((a+b) / 2)$ we have the midpoint inequality and its generalization and applications were considered in [6].

(ii) $c_{1}$ may be chosen depending on fixed $x$, e.g. $c_{1}=$ $-((x-a) /(b-a))$. In this case we get result which is an extension of the same result from [11] where the above inequality was proved with $L=\left\|f^{\prime \prime}\right\|_{\infty}$ for a class of functions with bounded second derivatives.

THEOREM 3 If $f^{\prime}$ is L-Lipschitizian on $[a, b]$, then for every $x \in[a, b]$ we have

$$
\begin{gathered}
\mid \int_{a}^{b} f(t) \mathrm{d} t-\frac{1}{2}\left[f(x)+\frac{f(a)+f(b)}{2}\right](b-a) \\
\quad+\frac{1}{2}(b-a)\left(x-\frac{a+b}{2}\right) f^{\prime}(x) \mid \\
\leq L\left(\frac{1}{3}\left|x-\frac{a+b}{2}\right|^{3}+\frac{(b-a)^{3}}{48}\right)
\end{gathered}
$$

Proof If we put $n=2$ in the first identity from Theorem 1 we have

$$
\begin{aligned}
f(x)= & \frac{1}{b-a} \int_{a}^{b} f(t) \mathrm{d} t+P_{1}\left(\frac{x-a}{b-a}\right)[f(b)-f(a)] \\
& +(b-a) P_{2}\left(\frac{x-a}{b-a}\right)\left[f^{\prime}(b)-f^{\prime}(a)\right]+(b-a) \alpha_{2} f^{\prime}(x) \\
& -(b-a) \int_{[a, b]} P_{2}^{*}\left(\frac{x-t}{b-a}\right) \mathrm{d} f^{\prime}(t),
\end{aligned}
$$

where

$$
P_{1}(t)=t+c_{1}, P_{2}(t)=\left(t^{2} / 2\right)+c_{1} t+c_{2}
$$

and

$$
\alpha_{2}=-\left((1 / 2)+c_{1}\right) .
$$

First we chose $c_{1}$ and $c_{2}$ such that $P_{1}((x-a) /(b-a))=0$ and $P_{2}((x-a) / \quad(b-a))=0$, i.e. $\quad c_{1}=-((x-a) /(b-a))$ and $c_{2}=$ $(1 / 2)((x-a) /(b-a))^{2}$. This gives $P_{1}(t)=t-((x-a) /(b-a))$, 
$P_{2}(t)=(1 / 2)(t-((x-a) /(b-a)))^{2} \quad$ and $\quad \alpha_{2}=(x-((a+b) / 2)) /$ $(b-a)$ so that we have

$$
\begin{aligned}
f(x)= & \frac{1}{b-a} \int_{a}^{b} f(t) \mathrm{d} t+\left(x-\frac{a+b}{2}\right) f^{\prime}(x) \\
& -(b-a) \int_{[a, b]} P_{2}^{*}\left(\frac{x-t}{b-a}\right) \mathrm{d} f^{\prime}(t) .
\end{aligned}
$$

Further we consider the identity (3.5) for $x=b$ with $P_{1}(t)$ and $P_{2}(t)$ replaced by $\tilde{P_{1}}(t)=t+\tilde{c_{1}}$ and $\tilde{P}_{2}(t)=\left(t^{2} / 2\right)+\tilde{c_{1}} t+\tilde{c_{2}}$, respectively, i.e.

$$
\begin{aligned}
f(b)= & \frac{1}{b-a} \int_{a}^{b} f(t) \mathrm{d} t+\tilde{P}_{1}(1)[f(b)-f(a)] \\
& +(b-a) \tilde{P}_{2}(1)\left[f^{\prime}(b)-f^{\prime}(a)\right] \\
& -(b-a)\left(\frac{1}{2}+\tilde{c_{1}}\right) f^{\prime}(x)-(b-a) \int_{[a, b]} \tilde{P}_{2}^{*}\left(\frac{b-t}{b-a}\right) \mathrm{d} f^{\prime}(t) .
\end{aligned}
$$

We chose $\tilde{c_{1}}$ and $\tilde{c_{2}}$ such that $(1 / 2)+\tilde{c_{1}}=0$ and $\tilde{P_{2}}(1)=0$, i.e. $\tilde{c_{1}}=-(1 / 2)$ and $\tilde{c_{2}}=0$. This gives $\tilde{P}_{1}(t)=t-(1 / 2), \quad \tilde{P_{2}}(t)=$ $(1 / 2) t(t-1)$ and

$$
\begin{aligned}
f(b)= & \frac{1}{b-a} \int_{a}^{b} f(t) \mathrm{d} t+\frac{1}{2}[f(b)-f(a)] \\
& -(b-a) \int_{[a, b]} \tilde{P_{2}^{*}}\left(\frac{b-t}{b-a}\right) \mathrm{d} f^{\prime}(t)
\end{aligned}
$$

or equivalently

$$
\frac{f(a)+f(b)}{2}=\frac{1}{b-a} \int_{a}^{b} f(t) \mathrm{d} t-(b-a) \int_{[a, b]} \tilde{P}_{2}^{*}\left(\frac{b-t}{b-a}\right) \mathrm{d} f^{\prime}(t) .
$$


If we multiply (3.6) and (3.7) with (1/2) and then add them up, we get the identity

$$
\begin{gathered}
\frac{1}{b-a} \int_{a}^{b} f(t) \mathrm{d} t-\frac{1}{2}\left[f(x)+\frac{f(a)+f(b)}{2}\right]+\frac{1}{2}\left(x-\frac{a+b}{2}\right) f^{\prime}(x) \\
=\frac{b-a}{2} \int_{[a, b]}\left[P_{2}^{*}\left(\frac{x-t}{b-a}\right)+\tilde{P}_{2}^{*}\left(\frac{b-t}{b-a}\right)\right] \mathrm{d} f^{\prime}(t) .
\end{gathered}
$$

If we denote the left hand side of (3.8) by $R(x)$, then using the estimate (3.3) we get

$$
\begin{aligned}
|R(x)| & \leq \frac{L(b-a)}{2} \int_{a}^{b}\left|P_{2}^{*}\left(\frac{x-t}{b-a}\right)+\tilde{P}_{2}^{*}\left(\frac{b-t}{b-a}\right)\right| \mathrm{d} t \\
& =\frac{L}{b-a}\left[\frac{1}{3}\left|x-\frac{a+b}{2}\right|^{3}+\frac{(b-a)^{3}}{48}\right] .
\end{aligned}
$$

Multiplying the above inequality by $b-a>0$ we get (3.4). (The equality case in the above expression can be done by elementary but rather long calculation and we omit the details).

Remark 3 When $f$ is a twice differentiable function with bounded and integrable second derivative, the inequality (3.4) holds with $L=\left\|f^{\prime \prime}\right\|_{\infty}$. So this inequality is a correction and in the same time an extension of the main result from [14]. Namely it is easy to see that

$$
P_{2}^{*}\left(\frac{x-t}{b-a}\right)+\tilde{P}_{2}^{*}\left(\frac{b-t}{b-a}\right)=\frac{1}{(b-a)^{2}} K(x, t),
$$

where

$$
K(x, t)= \begin{cases}(t-a)\left(t-\frac{(a+b)}{2}\right), & \text { for } t \in[a, x], \\ (t-b)\left(t-\frac{(a+b)}{2}\right), & \text { for } t \in(x, b]\end{cases}
$$


and for twice differentiable function $f$ with integrable second derivative the identity (3.8) multiplied by $b-a$ reduces to

$$
\begin{aligned}
& \int_{a}^{b} f(t) \mathrm{d} t-\frac{1}{2}\left[f(x)+\frac{f(a)+f(b)}{2}\right](b-a)+\frac{1}{2}(b-a)\left(x-\frac{a+b}{2}\right) f^{\prime}(x) \\
&=\frac{1}{2} \int_{a}^{b} K(x, t) f^{\prime \prime}(t) \mathrm{d} t .
\end{aligned}
$$

In [14] the incorrect version of the identity (3.11), with $-(b-$ $a)(x-(a+b) / 2) f^{\prime}(x)$ in place of $(1 / 2)(b-a)(x-(a+b) / 2) f^{\prime}(x)$, was obtained as a basic result.

THEOREM 4 Let $f:[a, b] \rightarrow \mathbf{R}$ be such that $f^{(n-1)}$ is a continuous function of bounded variation on $[a, b]$ for some $n \geq 1$. Then

$$
\begin{gathered}
\left|f(x)-\frac{1}{b-a} \int_{a}^{b} f(t) \mathrm{d} t-\tilde{T}_{n-1}(x)-\tau_{n}(x)\right| \\
\leq(b-a)^{n-1} \max _{t \in[0,1]}\left|P_{n}(t)-P_{n}\left(\frac{x-a}{b-a}\right)\right| V_{a}^{b}\left(f^{(n-1)}\right), \\
\quad\left|f(x)-\frac{1}{b-a} \int_{a}^{b} f(t) \mathrm{d} t-\tilde{T}_{n}(x)-\tau_{n}(x)\right| \\
\leq(b-a)^{n-1} \max _{t \in[0,1]}\left|P_{n}(t)\right| V_{a}^{b}\left(f^{(n-1)}\right)
\end{gathered}
$$

for every $x \in[a, b]$, where $V_{a}^{b}\left(f^{(n-1)}\right)$ is the total variation of $f^{(n-1)}$ on $[a, b]$.

Proof If $F:[a, b] \rightarrow \mathbf{R}$ is bounded and the Stieltjes integral $\int_{[a, b]} F(t) \mathrm{d} f^{(n-1)}(t)$ exists, then

$$
\left|\int_{[a, b]} F(t) \mathrm{d} f^{(n-1)}(t)\right| \leq \max _{t \in[a, b]}|F(t)| \cdot V_{a}^{b}\left(f^{(n-1)}\right) .
$$


Let us apply this estimation to the second formula of Theorem 1. We have

$$
\left|f(x)-\frac{1}{b-a} \int_{a}^{b} f(t) \mathrm{d} t-\tilde{T}_{n-1}(x)-\tau_{n}(x)\right|=\left|\tilde{R}_{n}^{2}(x)\right|,
$$

and

$$
\begin{aligned}
\left|\tilde{R}_{n}^{2}(x)\right| & =\left|-(b-a)^{n-1} \int_{a}^{b}\left[P_{n}^{*}\left(\frac{x-t}{b-a}\right)-P_{n}\left(\frac{x-a}{b-a}\right)\right] \mathrm{d} f^{(n-1)}(t)\right| \\
& \leq(b-a)^{n-1} \max _{t \in[a, b]}\left|P_{n}^{*}\left(\frac{x-t}{b-a}\right)-P_{n}\left(\frac{x-a}{b-a}\right)\right| V_{a}^{b}\left(f^{(n-1)}\right) \\
& =(b-a)^{n-1} \max _{t \in[0,1]}\left|P_{n}(t)-P_{n}\left(\frac{x-a}{b-a}\right)\right| V_{a}^{b}\left(f^{(n-1)}\right),
\end{aligned}
$$

which proves our first assertion. Similarly we prove the second one.

Remark 4 The first inequality for $n=1$ was proved in [9] (see also [7] and [17]). The second one with $n=1$ and $c_{1}=-((x-a) /(b-a))$ was proved in [9]. For $n=2$ and $c_{1}=-((x-a) /(b-a))$ in first inequality we have result which is an extension of the result from [12] with $V_{a}^{b}\left(f^{\prime}\right)=\left\|f^{\prime \prime}\right\|_{1}$ for a class of functions $f$ such that $f^{\prime \prime} \in L_{1}(a, b)$.

THEOREM 5 If $f^{\prime}$ is a continuous function of bounded variation on $[a, b]$ then

$$
\begin{aligned}
& \left|\int_{a}^{b} f(t) \mathrm{d} t-\frac{1}{2}\left[f(x)+\frac{f(a)+f(b)}{2}\right](b-a)+\frac{1}{2}(b-a)\left(x-\frac{a+b}{2}\right) f^{\prime}(x)\right| \\
& \leq \frac{V_{a}^{b}\left(f^{\prime}\right)}{2}\left\{\begin{array}{l}
\left|x-\frac{a+b}{2}\right|^{2}+\frac{(b-a)}{2}\left|x-\frac{a+b}{2}\right| \\
\text { for } x \in\left[a, \frac{1+\sqrt{2}}{4} a+\frac{3-\sqrt{2}}{4} b\right] \cup\left[\frac{3-\sqrt{2}}{4} a+\frac{1+\sqrt{2}}{4} b, b\right] \\
\frac{(b-a)^{2}}{16}, \\
\text { for } x \in\left(\frac{1+\sqrt{2}}{4} a+\frac{3-\sqrt{2}}{4} b, \frac{3-\sqrt{2}}{4} a+\frac{1+\sqrt{2}}{4} b\right)
\end{array}\right.
\end{aligned}
$$


Proof Using the estimate (3.12) we get from (3.8) and (3.9)

$$
\begin{aligned}
|R(x)| & \leq \frac{V_{a}^{b}\left(f^{\prime}\right)(b-a)}{2} \sup _{t \in[a, b]}\left|P_{2}^{*}\left(\frac{x-t}{b-a}\right)+\tilde{P}_{2}^{*}\left(\frac{b-t}{b-a}\right)\right| \\
& =\frac{V_{a}^{b}\left(f^{\prime}\right)}{2(b-a)} \sup _{t \in[a, b]}|K(x, t)|,
\end{aligned}
$$

where $R(x)$ is the left hand side of (3.8) and $K(x, t)$ is defined by (3.10). Further, by a simple calculation we get

$$
\begin{aligned}
& \sup _{t \in[a, b]}|K(x, t)|=\max \left[\frac{(b-a)^{2}}{16},\left|x-\frac{a+b}{2}\right|^{2}+\frac{b-a}{2}\left|x-\frac{a+b}{2}\right|\right\} \\
& =\left\{\begin{array}{l}
\left|x-\frac{a+b}{2}\right|^{2}+\frac{(b-a)}{2}\left|x-\frac{a+b}{2}\right|, \\
\text { for } x \in\left[a, \frac{1+\sqrt{2}}{4} a+\frac{3-\sqrt{2}}{4} b\right] \cup\left[\frac{3-\sqrt{2}}{4} a+\frac{1+\sqrt{2}}{4} b\right. \\
\frac{(b-a)^{2}}{16}, \\
\text { for } x \in\left(\frac{1+\sqrt{2}}{4} a+\frac{3-\sqrt{2}}{4} b, \frac{3-\sqrt{2}}{4} a+\frac{1+\sqrt{2}}{4} b\right) .
\end{array}\right.
\end{aligned}
$$

Substituting this in (3.13) and then multiplying by $b-a$, we get proposed inequality.

Remark 5 When $f$ is a twice differentiable function with integrable second derivative such that $f^{\prime \prime} \in L_{1}(a, b)$, the inequality proved in the above theorem holds with $V_{a}^{b}\left(f^{\prime}\right)$ replaced by $\left\|f^{\prime \prime}\right\|_{1}$. Therefore this inequality can be regarded as a double correction and, in the same time, as an extension of the analogous result from [15] for a class of functions $f$ with $f^{\prime \prime} \in L_{1}(a, b)$. The first correction is related to the expression within the absolute value sign at the left hand side (the same as in Remark 3$)$, while the second one is related to the obviously incorrect equality $\|K(x, \cdot)\|_{\infty}=\sup _{t \in[a, b]}|K(x, t)|=\left((b-a)^{2} / 4\right)$ which is stated and used in [15]. 
THEOREM 6 Let $f:[a, b] \rightarrow \mathbf{R}$ be such that $f^{(n)}$ is R-integrable and $f^{(n)} \in L_{p}[a, b]$ for some $n \geq 1$ and $1<p<\infty$. Then

$$
\begin{aligned}
& \left|f(x)-\frac{1}{b-a} \int_{a}^{b} f(t) \mathrm{d} t-\tilde{T}_{n-1}(x)-\tau_{n}(x)\right| \\
& \quad \leq(b-a)^{n-1+1 / q}\left(\int_{0}^{1}\left|P_{n}(t)-P_{n}\left(\frac{x-a}{b-a}\right)\right|^{q} \mathrm{~d} t\right)^{1 / q}\left\|f^{(n)}\right\|_{p} \\
& \left|f(x)-\frac{1}{b-a} \int_{a}^{b} f(t) \mathrm{d} t-\tilde{T}_{n}(x)-\tau_{n}(x)\right| \\
& \quad \leq(b-a)^{n-1+1 / q}\left(\int_{0}^{1}\left|P_{n}(t)\right|^{q} \mathrm{~d} t\right)^{1 / q}\left\|f^{(n)}\right\|_{p}
\end{aligned}
$$

for every $x \in[a, b]$, where $1 / p+1 / q=1$.

Proof Since $f^{(n)}$ is $R$-integrable, the integrals which occur in the expressions for $\tilde{R}_{n}^{1}(x)$ and $\tilde{R}_{n}^{2}(x)$ are the usual Riemann integrals with $\mathrm{d} f^{(n-1)}(t)$ replaced with $f^{(n)}(t) \mathrm{d} t$. So by applying the Hölder inequality we get from (2.6)

$$
\begin{aligned}
\mid f(x) & -\frac{1}{b-a} \int_{a}^{b} f(t) \mathrm{d} t-\tilde{T}_{n-1}(x)-\tau_{n}(x) \mid \\
& \leq(b-a)^{n-1} \int_{a}^{b}\left|P_{n}^{*}\left(\frac{x-t}{b-a}\right)-P_{n}\left(\frac{x-a}{b-a}\right)\right| f^{(n)}(t) \mid \mathrm{d} t \\
& \leq(b-a)^{n-1}\left(\int_{a}^{b}\left|P_{n}^{*}\left(\frac{x-t}{b-a}\right)-P_{n}\left(\frac{x-a}{b-a}\right)\right|^{q} \mathrm{~d} t\right)^{1 / q}\left\|f^{(n)}\right\|_{p} \\
& =(b-a)^{n-1+1 / q}\left(\int_{0}^{1}\left|P_{n}(t)-P_{n}\left(\frac{x-a}{b-a}\right)\right|^{q} \mathrm{~d} t\right)^{1 / q}\left\|f^{(n)}\right\|_{p}
\end{aligned}
$$

which proves the first stated inequality. The second one follows from (2.5) by the similar argument.

Remark 6 This first inequality of the theorem above for $n=1$ was proved by A. M. Fink [18] (see also [8] and [17]). 
THEOREM 7 If $f^{\prime \prime}$ is $R$-integrable and $f^{\prime \prime} \in L_{p}[a, b]$, for $1<p<\infty$, then

$$
\begin{aligned}
& \left|\int_{a}^{b} f(t) \mathrm{d} t-\frac{1}{2}\left[f(x)+\frac{f(a)+f(b)}{2}\right](b-a)+\frac{1}{2}\left(x-\frac{a+b}{2}\right) f^{\prime}(x)\right| \\
& \leq \frac{1}{2}\left(\frac{b-a}{2}\right)^{2+(1 / q)}\left\|f^{\prime}\right\|_{p} \\
& \quad \times\left\{\begin{array}{l}
{\left[B(q+1, q+1)+B_{x_{1}}(q+1, q+1)+\Psi_{x_{2}}(q+1, q+1)\right]^{(1 / q)}} \\
\text { for } x \in\left[a, \frac{a+b}{2}\right], \\
{\left[B(q+1, q+1)+B_{x_{3}}(q+1, q+1)+B_{x_{4}}(q+1, q+1)\right]^{(1 / q)}} \\
\text { for } x \in\left[\frac{a+b}{2}, b\right],
\end{array}\right.
\end{aligned}
$$

where $(1 / p)+(1 / q)=1, p>1, q>1$, and $B(\cdot, \cdot)$ and $B_{r}(\cdot, \cdot)$ are the Beta and the incomplete Beta function of Euler given by

$$
\begin{gathered}
B(l, s)=\int_{0}^{l} t^{l-1}(1-t)^{s-1} \mathrm{~d} t, \quad l, s>0, \quad B_{r}(l, s)=\int_{0}^{r} t^{l-1}(1-t)^{s-1} \mathrm{~d} t . \\
\Psi_{r}(l, s)=\int_{0}^{r} t^{l-1}(1+t)^{s-1} \mathrm{~d} t
\end{gathered}
$$

is a real positive valued integral, $x_{1}=(2(x-a) /(b-a)), x_{2}=1-x_{1}$, $x_{3}=x_{1}-1, x_{4}=2-x_{1}$.

Proof Assuming the correction of the expression at the left hand side as in the Remark 3, this inequality was proved in [15].

Remark 7 For $n=1$ and $c_{1}=-(x-a / b-a)$ we get the inequality which is a result from [18].

\section{References}

[1] Abramowitz, M. and Stegun, I. A. (Eds) (1965) Handbook of Mathematical Functions with Formulae,Graphs and Mathematical Tables, 4th printing, Applied Math.Series 55 (National Bureau of Standards, Washington).

[2] Berezin, I. S. and Zhidkov, N. P. (1965) Computing methods, Vol. I (Pergamon press, Oxford). 
[3] Krylov, V. I. (1962) Approximate Calculation of Integrals (Macmillan, New YorkLondon).

[4] Dedić, L. J., Matić, M. and Pečarić, J. (2000) “On generalizations of Ostrowski inequality via some Euler-type identities", Math. Inequal. \& Appl. 3(3), 337-353.

[5] Dedić, L. J., Matić, M. and Pečarić, J. (2001) "On Euler trapezoid formulae”, Applied Mathematics and Computation 123, 37-62.

[6] Dedić, L. J., Matić, M. and Pečarić, J. "On Euler mid-point formulae", ANZIAM J. (accepted).

[7] Dragomir, S. S. and Wang, S. (1997) "A new inequality of Ostrowski's type in $L_{1}$ norm and applications to some special means and to some numerical quadrature rules", Tamkang Journal of Mathematics 3(28), 239-244.

[8] Dragomir, S. S. and Wang, S. (1998) "A new inequality of Ostrowski's type in $L_{p}$ norm and applications to some special means and to some numerical quadrature rules", Indian Journal of Mathematics 40(3), 299-304.

[9] Dragomir, S. S. (1999) "On the Ostrowski inequality for mappings with bounded variation and applications", Bull. Austral. Math. Soc. 60, 495-508.

[10] Dragomir, S. S. (1999) "On the Ostrowski inequality for Lipschitzian mappings and applications", Computers and Mathematics with Applications 38, 33-37.

[11] Cerone, P., Dragomir, S. S. and Roumeliotis, J. (1999) "An inequality of Ostrowski type for mappings whose second derivatives are bounded and applications", East Asian Math. J. 15(1), 1-9.

[12] Cerone, P., Dragomir, S. S. and Roumeliotis, J. (1999) "An inequality of Ostrowski type for mappings whose second derivatives belong to $L_{1}(a, b)$ and applications", Honam Mathematical J. 21(1), 127-137.

[13] Dragomir, S. S. and Barnett, N. S. (1999) "On the Ostrowski inequality for mappings with bounded variation and applications", J. Indian Math. Soc. (N.S.) 66(1-4), 237-245.

[14] Dragomir, S. S. and Sofo, A. (1999) "An integral inequality for twice differentiable mappings and applications", RGMIA Res. Rep. Coll. 2(2).

[15] Dragomir, S. S. and Sofo, A. (2000) "An inequality of Ostrowski for twice differentiable mappings in terms of the $L_{p}$ norm and applications", RGMIA Res. Rep. Coll. 3(1).

[16] Dragomir, S. S., Agarwal, R. P. and Cerone, P. (2000) "On Simpson's inequality and applications", Journal Inequalities and Applications 5, 533-579.

[17] Dragomir, S. S., Agarwal, R. P. and Barnett, N. S. (2000) "Inequalities for Beta and Gamma functions via some classical and new inequalities", Journal of Inequalities and Applications 5, 103-165.

[18] Fink, A. M. (1992) "Bounds of the deviation of a function from its averages", Czechoslovak Math. J. 42(117), 289-310. 